\title{
Nuclear reactions in copper induced by protons from a petawatt laser-foil interaction
}

\author{
J. M. Yang ${ }^{\text {a) }}$ \\ Department of Physics, University of Strathclyde, Glasgow G4 ONG, United Kingdom and Research Center \\ of Laser Fusion, P.O. Box 919-986, Mianyang 621900, People's Republic of China \\ P. McKenna \\ Department of Physics, University of Strathclyde, Glasgow G4 ONG, United Kingdom
}

K. W. D. Ledingham

Department of Physics, University of Strathclyde, Glasgow G4 ONG, United Kingdom and AWE plc, Aldermaston, Reading RG7 4PR, United Kingdom

T. McCanny, S. Shimizu, and L. Robson

Department of Physics, University of Strathclyde, Glasgow G4 ONG, United Kingdom

R. J. Clarke, D. Neely, and P. A. Norreys

Central Laser Facility, Rutherford Appleton Laboratory, Chilton, Didcot, Oxon OX11 OQX, United Kingdom

M.-S. Wei, K. Krushelnick, P. Nilson, and S. P. D. Mangles

Blackett Laboratory, Imperial College, Prince Consort Road, London SW7 2BZ, United Kingdom

\author{
R. P. Singhal \\ Department of Physics and Astronomy, University of Glasgow, Glasgow G12 8QQ, United Kingdom
}

(Received 24 October 2003; accepted 4 December 2003)

\begin{abstract}
High-intensity $\left(>10^{19} \mathrm{~W} \mathrm{~cm}^{-2}\right.$ ) laser-plasma interactions have been shown to produce large quantities of protons with energies up to several tens of $\mathrm{MeV}$. A range of laser-driven proton-induced reactions in copper have been investigated and the observed reactions quantified. The energy spectrum of the accelerated protons was determined from the reactions in a single thin copper foil and found to be in agreement with that deduced from $(p, n)$ reactions measured in a stack of copper foils. The potential applications of this diagnostic technique are discussed. (C) 2004 American Institute of Physics. [DOI: 10.1063/1.1645314]
\end{abstract}

High intensity laser-plasma interactions at intensities greater than $10^{19} \mathrm{~W} / \mathrm{cm}^{2}$ have been shown to lead to fast electron acceleration to energies larger than $200 \mathrm{MeV}^{1} \gamma$ radiation and energetic ions including a few tens of $\mathrm{MeV}$ protons $^{2-4}$ and several hundred MeV heavy ions. ${ }^{5,6}$ There are many potential applications of these energetic particles and high energy $\gamma$ rays. For example, in the past few years multi-MeV proton beams, generated from intense laserplasma interactions, have been used to produce short-lived positron emitting isotopes in low-Z materials such as ${ }^{11} \mathrm{~B}$ and $\mathrm{H}_{2}{ }^{18} \mathrm{O}$ via $(p, n)$ reactions. ${ }^{7-9}$ Multi-MeV proton beams have also been used as a particle probe to measure electric charging of microscopic targets irradiated by a laser at an intensity of $10^{19} \mathrm{~W} / \mathrm{cm}^{2} .{ }^{10}$ The proton energy spectrum is often diagnosed by measuring $\beta^{+}$decay from induced ${ }^{63} \mathrm{Cu}(p, n){ }^{63} \mathrm{Zn}$ reactions in copper stacks, using a NaI detectors based coincidence counting system. ${ }^{5,8,11}$ With increasing laser intensity, protons are produced with much higher energies and can induce reactions with higher $Q$ values.

This letter reports a range of observed reactions in copper, induced by proton beams from a petawatt laser-solid interaction. By measuring the reactions induced in a single thin $\mathrm{Cu}$ foil a method of diagnosing the proton energy spectrum produced in an experiment of this type is highlighted.

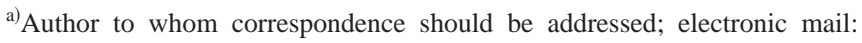
j.yang@phys.strath.ac.uk
}

This technique is less invasive to the proton beams and therefore could be used in conjunction with potential applications of the proton beams.

The experiment was carried out on the new petawatt arm of the VULCAN Nd:Glass laser at the Rutherford Appleton Laboratory, U.K. The $60 \mathrm{~cm}$ beam was focused to a $\sim 7.0$ $\mu \mathrm{m}$-diam spot using a $1.8 \mathrm{~m}$ focal length off-axis parabolic mirror, in a vacuum chamber evacuated to $\sim 10^{-4}$ mbar. The average pulse duration was $\sim 750 \mathrm{fs}$ and the energy on target was $\sim 170 \mathrm{~J}$ with a corresponding intensity of $\sim 2$ $\times 10^{20} \mathrm{~W} \mathrm{~cm}^{-2}$. A $10-\mu \mathrm{m}$-thick aluminum foil was irradiated by the $p$-polarized laser beam at an angle of $45^{\circ}$ to generate multi-MeV proton beams from both sides of the target. A stack of copper foils was positioned at both sides of the target along the target normal direction at a distance of 38 $\mathrm{mm}$. The size of the copper pieces in each stack was $50 \times 50$ $\mathrm{mm}$ with the first two pieces $100 \mu \mathrm{m}$ thick, the next two pieces $250 \mu \mathrm{m}$ thick, two pieces $500 \mu \mathrm{m}$ thick and finally two pieces of 1-mm-thick $\mathrm{Cu}$ positioned at the back of the stack (furthest from the target). The copper samples have two isotopes ${ }^{63} \mathrm{Cu}(69.17 \%)$ and ${ }^{65} \mathrm{Cu}(30.83 \%)$.

The $\mathrm{Cu}$ foils in the stacks were exposed to the proton beams from the laser-irradiated target and the production of various isotopes was observed in the foils. The front foil (100 $\mu \mathrm{m}$ thick) in each copper stack was analyzed before and after the laser shot using two well-shielded germanium detectors (CANBERRA GX3518). The isotopes and corresponding reactions in the samples were identified based on 


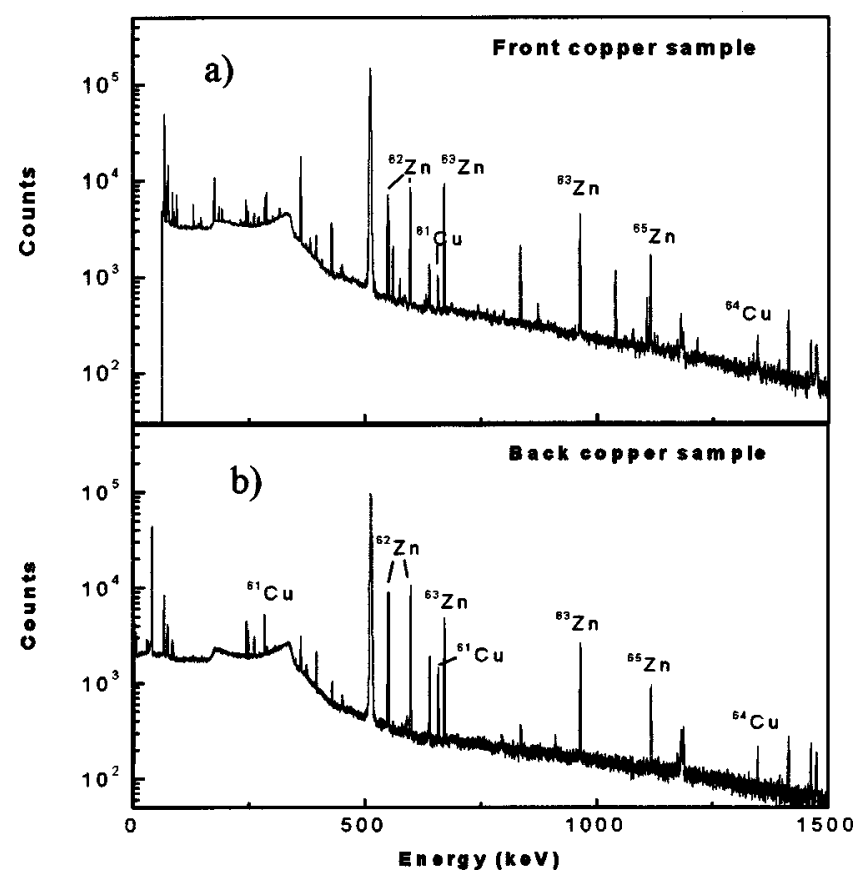

FIG. 1. The measured gamma spectra from the proton-irradiated copper foils. (a) Spectrum for front copper sample; (b) spectrum for back copper sample. The main peaks for the nuclei have been labeled in the spectra. Characteristic lines of some nuclei produced by heavy ion reactions have also been observed, especially for the front copper sample, which will be discussed elsewhere.

their emitted $\gamma$-ray energies, intensities, and half lives. The detection efficiencies of the two germanium detectors were calibrated using known sources of ${ }^{152} \mathrm{Eu},{ }^{57} \mathrm{Co},{ }^{22} \mathrm{Na},{ }^{137} \mathrm{Cs}$, and ${ }^{60} \mathrm{Co}$ to facilitate absolute numbers of laser induced reactions to be determined. The activity from the ${ }^{63} \mathrm{Cu}(p, n){ }^{63} \mathrm{Zn}$ reaction in each piece of copper in the stacks was measured using the $3 \mathrm{in} . \times 3$ in. NaI coincidence system, to obtain the proton spectra. The detection efficiency of the $\mathrm{NaI}$ coincidence system has also been calibrated using the ${ }^{22} \mathrm{Na}$ source.

Parts of the $\gamma$-ray spectra for the first $\mathrm{Cu}$ foil in both the front and rear samples, measured with the germanium detectors, are shown in Fig. 1. Characteristic $\gamma$-ray lines of the nuclei ${ }^{62} \mathrm{Zn},{ }^{63} \mathrm{Zn},{ }^{65} \mathrm{Zn},{ }^{61} \mathrm{Cu}$, and ${ }^{64} \mathrm{Cu}$ have been observed in both samples. These nuclei have mainly been generated through the proton-induced reactions ${ }^{63} \mathrm{Cu}(p, 2 n){ }^{62} \mathrm{Zn}$, ${ }^{63} \mathrm{Cu}(p, n){ }^{63} \mathrm{Zn},{ }^{65} \mathrm{Cu}(p, n){ }^{65} \mathrm{Zn},{ }^{63} \mathrm{Cu}(p, p+2 n){ }^{61} \mathrm{Cu}$ and ${ }^{65} \mathrm{Cu}(p, p+n){ }^{64} \mathrm{Cu}$, respectively. ${ }^{61} \mathrm{Cu}$ and ${ }^{64} \mathrm{Cu}$ may also be produced in ${ }^{63} \mathrm{Cu}(\gamma, 2 n){ }^{61} \mathrm{Cu}$ and ${ }^{65} \mathrm{Cu}(\gamma, n){ }^{64} \mathrm{Cu}$ reactions. However, in $10 \mu \mathrm{m} \mathrm{Al}$ primary target, the production of bremsstrahlung $\gamma$ rays is small and $\gamma$ induced reactions will

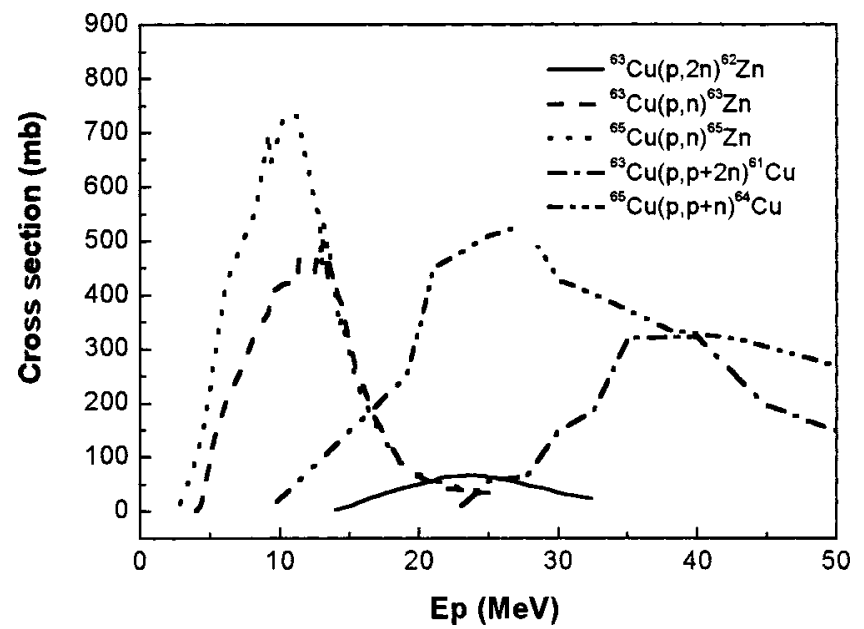

FIG. 2. The cross sections (see Ref. 12) for the observed reactions as a function of proton energy $E_{p}$.

make a negligible contribution to the observed yields. The numbers of all the observed nuclei in this shot have been calculated from the measured characteristic line intensities after taking into account the detection efficiency of the germanium detector, the decay branching ratio, $\gamma$-ray emitting possibilities, and the half lives of the isotopes. The numbers of each nuclei are listed in Table I along with the corresponding proton-induced reactions and the peak value of the cross sections of these reactions. The observed reactions have quite different $Q$ values and cross-section peak energies, ${ }^{12}$ e.g., the cross section for ${ }^{63} \mathrm{Cu}(p, n){ }^{63} \mathrm{Zn}$ peaks at $10.9 \mathrm{MeV}$, whereas the maximum for the ${ }^{63} \mathrm{Cu}(p, p+2 n){ }^{61} \mathrm{Cu}$ reaction is at $40 \mathrm{MeV}$, as shown in Fig. 2.

A code was developed to unfold the proton spectrum using the measured numbers of the observed isotopes. The first step involved the derivation of a response function. The number of nuclei produced by one proton with energy $E_{p}$ was calculated for each reaction using the published proton stopping range and cross section data. ${ }^{12}$ Then, starting with an arbitrary initial proton spectrum, the number of nuclei for each reaction was calculated by convoluting the spectrum with each response function. The ratio of the measured number of nuclei to calculated values was determined for each nucleus and the proton spectrum was adjusted repeatedly according to the ratio and its corresponding response energy region until the calculated numbers for all observed nuclei were consistent with the measured ones within the preset error. The proton spectra derived in this way at the front and back of the $\mathrm{Al}$ target foil are presented in Fig. 3.

The number of protons from the backside of the primary

TABLE I. Residual nuclei observed in the copper activation samples and corresponding proton-induced reactions.

\begin{tabular}{|c|c|c|c|c|c|c|}
\hline \multirow[b]{2}{*}{ Nuclei } & \multicolumn{2}{|c|}{ Observed number of nuclei } & \multirow[b]{2}{*}{ Reactions } & \multirow{2}{*}{$\begin{array}{c}Q \text { values } \\
(\mathrm{MeV})\end{array}$} & \multirow{2}{*}{$\begin{array}{l}\text { Peak cross } \\
\text { section } \sigma_{\text {peak }} \\
(\mathrm{mb})\end{array}$} & \multirow{2}{*}{$\begin{array}{l}\sigma_{\text {peak }} \\
\text { energy } \\
(\mathrm{MeV})\end{array}$} \\
\hline & Front side & Backside & & & & \\
\hline $62^{2 \mathrm{Zn}}$ & $(1.20 \pm 0.14) \times 10^{7}$ & $(1.65 \pm 0.40) \times 10^{7}$ & ${ }^{63} \mathrm{Cu}(p, 2 n){ }^{62} \mathrm{Zn}$ & -13.26 & 135 & 23.0 \\
\hline${ }^{63} \mathrm{Zn}$ & $(7.27 \pm 0.64) \times 10^{8}$ & $(4.45 \pm 0.45) \times 10^{8}$ & ${ }^{63} \mathrm{Cu}(p, n){ }^{63} \mathrm{Zn}$ & -4.149 & 500 & 13 \\
\hline${ }^{65} \mathrm{Zn}$ & $(7.88 \pm 0.82) \times 10^{8}$ & $(4.54 \pm 0.50) \times 10^{8}$ & ${ }^{65} \mathrm{Cu}(p, n){ }^{65} \mathrm{Zn}$ & -2.134 & 760 & 10.9 \\
\hline${ }^{61} \mathrm{Cu}$ & $(1.76 \pm 0.21) \times 10^{6}$ & $(5.56 \pm 0.43) \times 10^{6}$ & ${ }^{63} \mathrm{Cu}(p, p+2 n){ }^{61} \mathrm{Cu}$ & -19.74 & 323 & 40.0 \\
\hline${ }^{64} \mathrm{Cu}$ & $(6.5 \pm 1.2) \times 10^{7}$ & $(5.78 \pm 0.34) \times 10^{7}$ & ${ }^{65} \mathrm{Cu}(p, p+n){ }^{64} \mathrm{Cu}$ & -9.910 & 490 & 25.0 \\
\hline
\end{tabular}




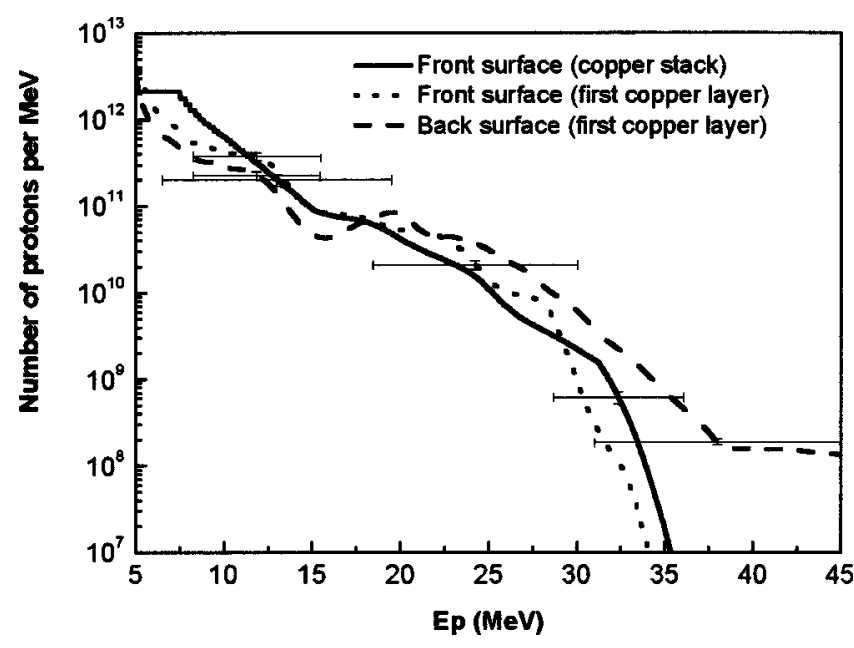

FIG. 3. Deduced proton spectra from the front and rear of the aluminum target, determined by proton-induced reactions in single thin layer of copper sample. The proton spectrum from the front side as also measured from ${ }^{63} \mathrm{Cu}(p, n){ }^{63} \mathrm{Zn}$ reactions in a stack of $\mathrm{Cu}$ foils is also shown for comparison. Typical error bars for the curves are shown.

target is smaller than that from the front side in the lower energy range $(<18 \mathrm{MeV})$, but is much larger in the higher energy range. The high-energy cutoff in the rear proton spectrum is $40 \mathrm{MeV}$, somewhat higher than that from the front proton spectrum $(33 \mathrm{MeV})$ in this shot. The conversion fractions of laser energy into proton beams greater than $5 \mathrm{MeV}$ were calculated from the proton spectra, which are $6.2 \%$ $(10.4 \mathrm{~J})$ for front proton beam and $4.3 \%(7.2 \mathrm{~J})$ for the rear one.

The proton spectrum at the front of the Al target was also measured with the copper stack technique for the same shot, as presented in Fig. 3. As shown, the proton spectrum determined from the induced reactions in the single $\mathrm{Cu}$ foil is in good agreement with that determined from ${ }^{63} \mathrm{Cu}(p, n){ }^{63} \mathrm{Zn}$ reaction in the copper stack, indicating that the technique can be employed in experiments of this type.

As the protons and other ions are produced through highly nonlinear processes in the plasma, there could exist a large shot-to-shot fluctuation in the proton spectra for similar laser intensities. Large fluctuations have also been observed in previous experiments ${ }^{13}$ and this could be a problem in the applications of the laser-generated proton beams unless the spectra can be monitored using noninvasive diagnostic techniques. The technique described in this work uses only a very thin layer of copper which can be inserted into the proton beam with minimal changes to the properties of the beam. In other words, the thin layer of copper could be used as a proton spectrum monitor without disrupting potential applications of the proton beam. A thinner copper layer has a smaller impact on the proton beam, but as a monitor of proton spectrum, thinner copper also has a lower sensitivity. The $\mathrm{Cu}$ thickness should be optimized according to the energies and intensities of the protons and the affordable impact of the monitor on the proton application.

In conclusion, proton beams generated from the interaction of a VULCAN petawatt laser pulse with an aluminum foil have been used to irradiate the copper samples and induce nuclear reactions. Five different proton-induced reactions in copper have been measured and used to develop a proton spectrum diagnostic technique. This technique, which employs a germanium detector, has an important advantage over the commonly used $\mathrm{Cu}$ foil stack technique, as it can be used in conjunction with other diagnostics or even with potential applications. As the cross-section peak energies of the observed proton-induced reactions in copper lie in the range of about 10-40 MeV, the technique is applicable to measure the proton energy spectrum in this energy range. It is also possible that the energy range can be extended by using a composition of copper and other material such as cobalt, zinc, and/or nickel in a single foil in which proton-induced reactions with much higher or lower $Q$ values can be observed.

The authors would like to acknowledge the expertise of the VULCAN operations team. J.M.Y. acknowledges support from the China Scholarship Council. P.McK. is supported by a Royal Society of Edinburgh/SEELLD research fellowship. S.S. acknowledges support from the Japan Society for the Promotion of Science. This work is funded by the EPSRC(UK).

${ }^{1}$ V. Malka, S. Fritzler, E. Lefebvre, F. Aleonard, F. Burgy, J.-P. Chambaret, J.-F. Chemin, K. Krushelnick, G. Malka, S. P. D. Mangles, Z. Najmudin, M. Pittman, J.-P. Rousseau, J.-N. Scheurer, B. Walton, and A. E. Dangor, Science 22, 1596 (2002).

${ }^{2}$ See, for example J. T. Mendonca, J. R. Davies, and M. Eloy, Meas. Sci. Technol. 12, 1801 (2001), and references therein.

${ }^{3}$ A. J. Mackinnon, M. Borghesi, S. Hatcher, M. H. Key, P. K. Patel, H. Campbell, A. Schiavi, R. Snavely, S. C. Wilks, and O. Willi, Phys. Rev. Lett. 86, 1769 (2001).

${ }^{4}$ S. Wilks, A. B. Longdon, T. E. Cowan, M. Roth, M. Singh, S. Hatchett, M. H. Key, D. Pennington, A. Mackinnon, and R. A. Snavely, Phys. Plasmas 8, $542(2001)$.

${ }^{5}$ E. L. Clark, K. Krushelnick, M. Zepf, F. N. Beg, M. Tatarakis, A. Machacek, M. I. K. Santala, I. Watts, P. A. Norreys, and A. E. Dangor, Phys. Rev. Lett. 85, 1654 (2000).

${ }^{6}$ P. McKenna, K. W. D. Ledingham, T. McCanny, R. P. Singhal, I. Spencer, M. Santala, F. N. Beg, A. E. Dangor, K. Krushelnick, M. Takarakis, M. S. Wei, E. L. Clark, R. J. Clarke, K. L. Lancaster, P. A. Norreys, K. Spohr, R. Chapman, and M. Zepf, Phys. Rev. Lett. 91, 075006 (2003).

${ }^{7}$ K. W. D. Ledingham, P. McKenna, and R. P. Singhal, Science 300, 1107 (2003).

${ }^{8}$ I. Spencer, K. W. D. Ledingham, R. P. Singhal, T. McCanny, P. McKenna, E. L. Clark, K. Krushelnick, M. Zepf, F. N. Beg, M. Tatarakis, A. E. Dangor, P. A. Norreys, R. J. Clarke, R. M. Allott, and I. N. Ross, Nucl. Instrum. Methods Phys. Res. B 183, 449 (2001).

${ }^{9}$ K. W. D. Ledingham, P. McKenna, T. McCanny, S. Shimizu, J. Yang, L. Robson, J. Zweit, J. M. Gillies, J. Bailey, G. N. Chimon, R. P. Singhal, M. S. Wei, S. Mangles, P. Nilson, K. Krushelnick, M. Zepf, R. J. Clarke, and P. A. Norreys (unpublished).

${ }^{10}$ M. Borghesi, D. H. Campbell, A. Schiavi, M. G. Haines, O. Willi, A. J. Mackinnon, P. Patel, L. A. Gizzi, M. Galimberti, R. J. Clarke, F. Pegoraro, H. Ruhl, and S. Bulanov, Phys. Plasmas 9, 2214 (2002).

${ }^{11}$ E. L. Clark, K. Krushelnick, J. R. Davies, M. Zepf, M. Tatarakis, F. N. Beg, A. Machacek, P. A. Norreys, M. I. K. Santala, I. Watts, and A. E. Dangor, Phys. Rev. Lett. 84, 670 (2000).

${ }^{12}$ EXFOR cross-section data online at http://www.nea.fr/html/dbdata/x4/ welcome.html

${ }^{13}$ M. I. K. Santala, M. Zepf, F. N. Beg, E. L. Clark, A. E. Dangor, K. Krushelnick, M. Tatarakis, I. Watts, K. W. D. Ledingham, T. McCanny, I. Spencer, A. C. Machacek, R. Allot, R. J. Clarke, and P. A. Norreys, Appl. Phys. Lett. 78, 19 (2001). 